\title{
Cataclysmic variables - a research laboratory for accretion processes
}

\section{Vojtěch Šimon*}

Astronomical Institute, The Czech Academy of Sciences, 25165 Ondřejov, Czech Republic

Czech Technical University in Prague, Faculty of Electrical Engineering, Prague, Czech

Republic

E-mail: simon@asu.cas.cz

\begin{abstract}
We show that cataclysmic variables (CVs) are important laboratories for investigation of accretion processes. It is important that a CV binary works as a system strongly variable with time. The character of the long-term activity (and operation of the emission processes) largely depend on the mass inflow into the lobe of the WD, and on the strength of the magnetic field of this WD. Strong structural changes of the emitting regions with time often accompany the transitions between the states of activity. Cycles of this activity (often on timescale of months to years) are common. Episodic events are the common properties of this activity which often consists of the discrete levels of intensity and/or discrete phenomena (e.g. outbursts, low state episodes). We also show that classical nova explosion has the large consequences for the type of activity (and even its change) after returning to quiescence in some CVs. Investigation of interplay of various emitting regions (and emission processes) in various states of activity is very important.
\end{abstract}

Frontier Research in Astrophysics - II

23-28 May 2016

Mondello (Palermo), Italy

* Speaker. 


\section{Introduction}

Cataclysmic variables (CVs) are important laboratories for investigation of accretion processes. They are close binary systems (the typical orbital period $P_{\text {orb }}$ is several hours) in which matter transfers onto the white dwarf (WD) from its companion (see [46] for a review). The strength $B$ of the magnetic field of the WD strongly affects the flow and the structure of the transferring matter. Transfer of matter onto the WD in CVs causes strong activity in various spectral bands (from hard X-rays and sometimes even gamma-rays (e.g. $[25,26])$ to the optical, infrared, sometimes extending to the radio band (e.g. [21])).

\section{Mechanisms and processes for the long-term activity of CVs}

Various emission processes, dependent on the circumstances in a given $\mathrm{CV}$, can operate in the different spectral bands (e.g. [46]). This is reflected in the resulting spectral energy distribution (SED). The behavior of $\mathrm{X}$-ray emission in a given state of activity can vary from one $\mathrm{CV}$ to another (compare the objects e.g. in $[48,5,38]$ ).

These emission processes and their time variations cause a very large range of the optical luminosities of CVs (measured as the absolute magnitudes) (Fig. 1). The luminosity of a given CV can change significantly, but the total range of luminosities is mainly caused by the fact that several types of CVs exist. The luminosity of this emission can reach very high values in some events (e.g. outbursts) (e.g. [46]). The activity of CVs can be governed by the following processes:

- Changes of the mass transfer rate from the donor onto the $\mathrm{WD}, \dot{m}_{\mathrm{T}}$ (timescale: days, weeks, months, years). The luminosity of a CV strongly depends on $\dot{m}_{\mathrm{T}}$. Most X-rays are emitted via bremsstrahlung process from boundary layer (encircling the equator of the WD) or from the accretion columns onto the magnetic poles of the WD. Most of the optical emission comes from the accretion disk radiating thermal emission (if it exists). In the case of CVs with strongly magnetized WD ( $B>10^{7}$ Gauss), no accretion disk can be formed, and the energy is radiated mostly from the accretion column via cyclotron (optical band), bremsstrahlung (accretion column - hard X-rays), and thermal (soft X-rays - WD heated by the impact) $[11,23]$.

- Thermal-viscous instability of the accretion disk [40, 14] (timescale: days, weeks, months). It operates in CVs within a certain range of $\dot{m}_{\mathrm{T}}$. It triggers the outbursts in dwarf novae. They begin when the accretion disk switches from the cold to the hot state after accumulating a sufficient amount of matter. This causes a strong increase of the temperature and viscosity of the disk matter, resulting in its accretion onto the WD at a large rate $\dot{m}_{\text {acc }}$. The optical luminosity (thermal emission of the disk) increases, but the variations of X-ray luminosity (representing bremsstrahlung emission from boundary layer or the polar caps on the WD) are complicated and even largely differ from one dwarf nova to another (compare e.g. [4] and [5]). These complex X-ray changes can be attributed to the large structural changes of the emitting regions between outburst and quiescence [28]. Novalikes are CVs whose disks are mostly in the hot state. 


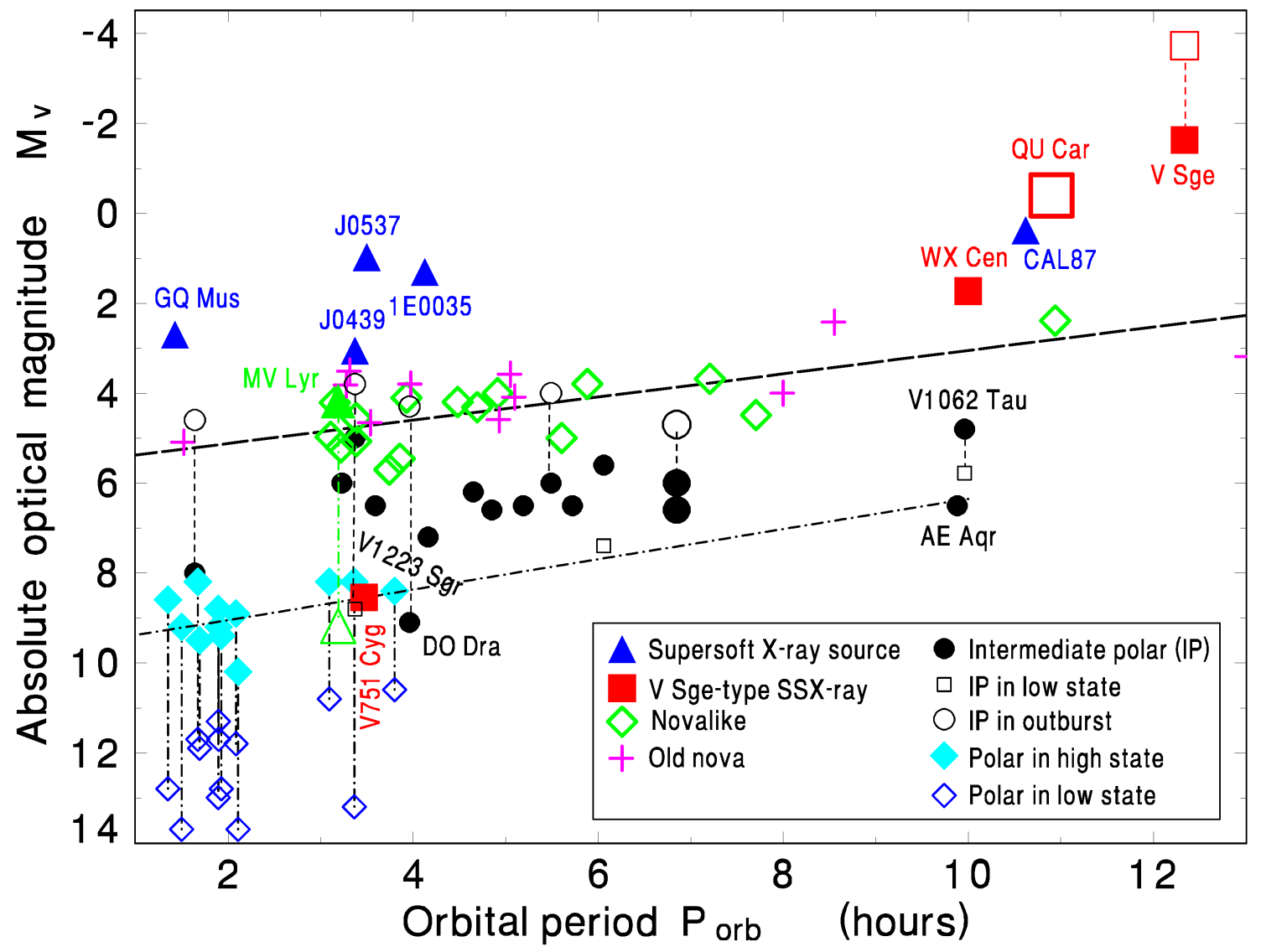

Figure 1: Absolute optical magnitudes of CVs of various types versus their $P_{\text {orb }}$. Outbursts and low states are marked by the empty circles and squares, respectively (most data are from [45, 46, 37]). GK Per and X Ser lie out of range because of their long $P_{\text {orb }}$. The relation between $P_{\text {orb }}$ and the peak brightness of outbursts of dwarf novae and their quiescent level are according to [45]. Most data are from [45, 46, 42, 37, 8].

- Hydrogen burning of the accreted matter on the WD (e.g. [49]): (a) Episodic: classical nova explosion (timescale: weeks, months); (b) Steady-state: supersoft X-ray sources (timescale: days, weeks, months). If the mass accretion rate onto the WD from the donor star is extremely high $\left(\sim 10^{-6} M_{\odot}\right.$ year $\left.^{-1}\right)$ [44], then both accretion and steady-state thermonuclear burning of the accreted matter on the surface of the WD are powerful energy sources. These $\mathrm{CV}$ binaries are called (quasi)persistent supersoft X-ray sources. Most of their X-ray luminosity comes from this burning (observed as a very hot atmosphere of the WD). A very intense X-ray emission and the luminosity even reaching the Eddington limit $\left(\sim 10^{38} \mathrm{erg} \mathrm{s}^{-1}\right)$ are produced [29].

A relation of the optical and X-ray luminosities of CVs in Fig. 2 shows that the steady-state thermonuclear burning of the accreted hydrogen on the WD is able to provide a much higher luminosity than accretion can do. 


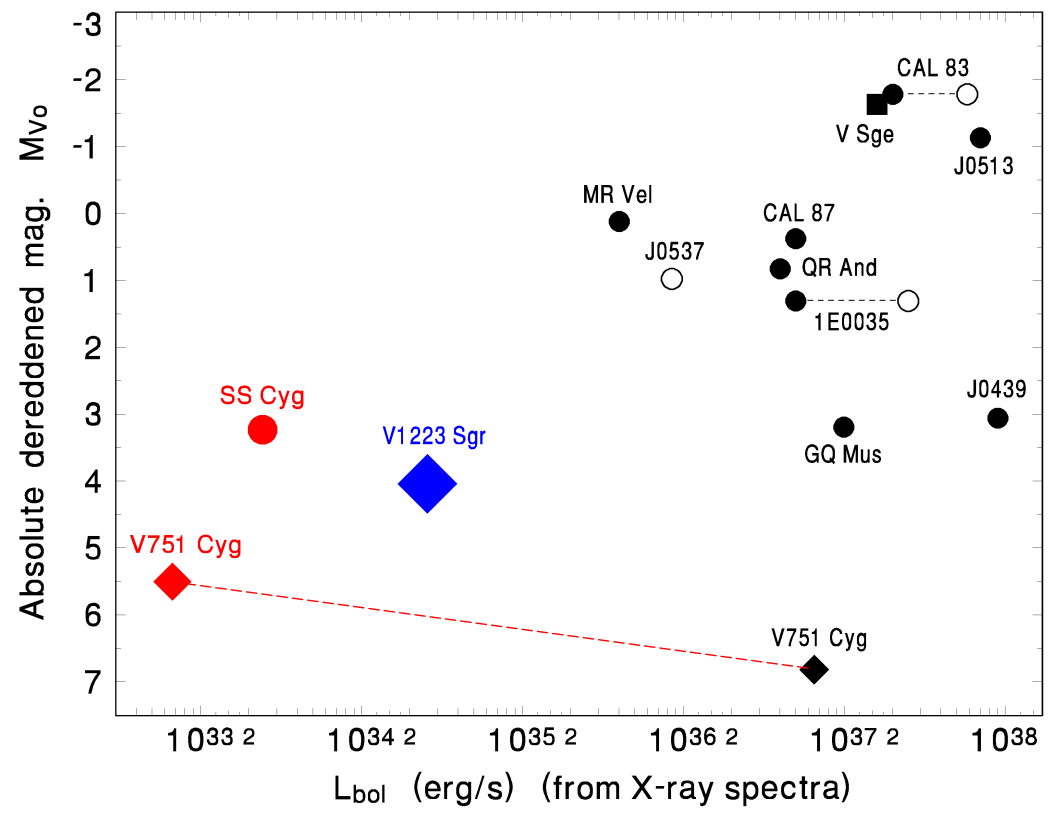

Figure 2: Relation of the optical and $\mathrm{X}$-ray luminosities of CVs of various types. Thermonuclear burning on the WD is able to provide a much higher luminosity than accretion can do. The luminosity is generated by accretion of matter for CVs with $L_{\mathrm{bol}}>10^{35} \mathrm{erg} \mathrm{s}^{-1}$. In the case of supersoft X-ray sources $\left(L_{\mathrm{bol}}>\right.$ $10^{35} \mathrm{erg} \mathrm{s}^{-1}$ ), thermonuclear hydrogen burning of the accreted mater largely contributes to the luminosity (filled symbols: a very hot atmosphere of the WD, open symbols: black-body fits). V751 Cyg became a supersoft X-ray source only in the optical low state. SS Cyg was in outburst, V1223 Sgr in the high state. Data sources: [24, 2, 27, 37].

\section{Investigation of the transition between $\mathrm{CV}$ types}

CVs are highly variable with time, and even different processes governing the activity can operate in a given system in various times. Outbursts of classical novae cause strong changes, with the consequences lasting for many years after returning to quiescence. This nova outburst especially influences the effective temperature of the WD in the following years after this event. Cooling of the WD after the thermo-nuclear explosion gives rise to evolution from novalike to dwarf nova.

\subsection{GK Persei}

The long-term activity of GK Per can serve as a very important example. This CV displays strong activity in quiescence after return to quiescence from the classical nova outburst. Its type of activity changed from classical nova to novalike, and finally to dwarf nova during several tens of years [33, 18]. Figure 3 shows a longer data series, extending to 2016. The dwarf nova outbursts appeared only when the thermal- viscous disk instability could operate since the cooled WD was already not able to irradiate the accretion disk sufficiently to keep it in the hot branch. This enabled the accretion disk to switch between the hot and cool states, as modeled by [34]. 


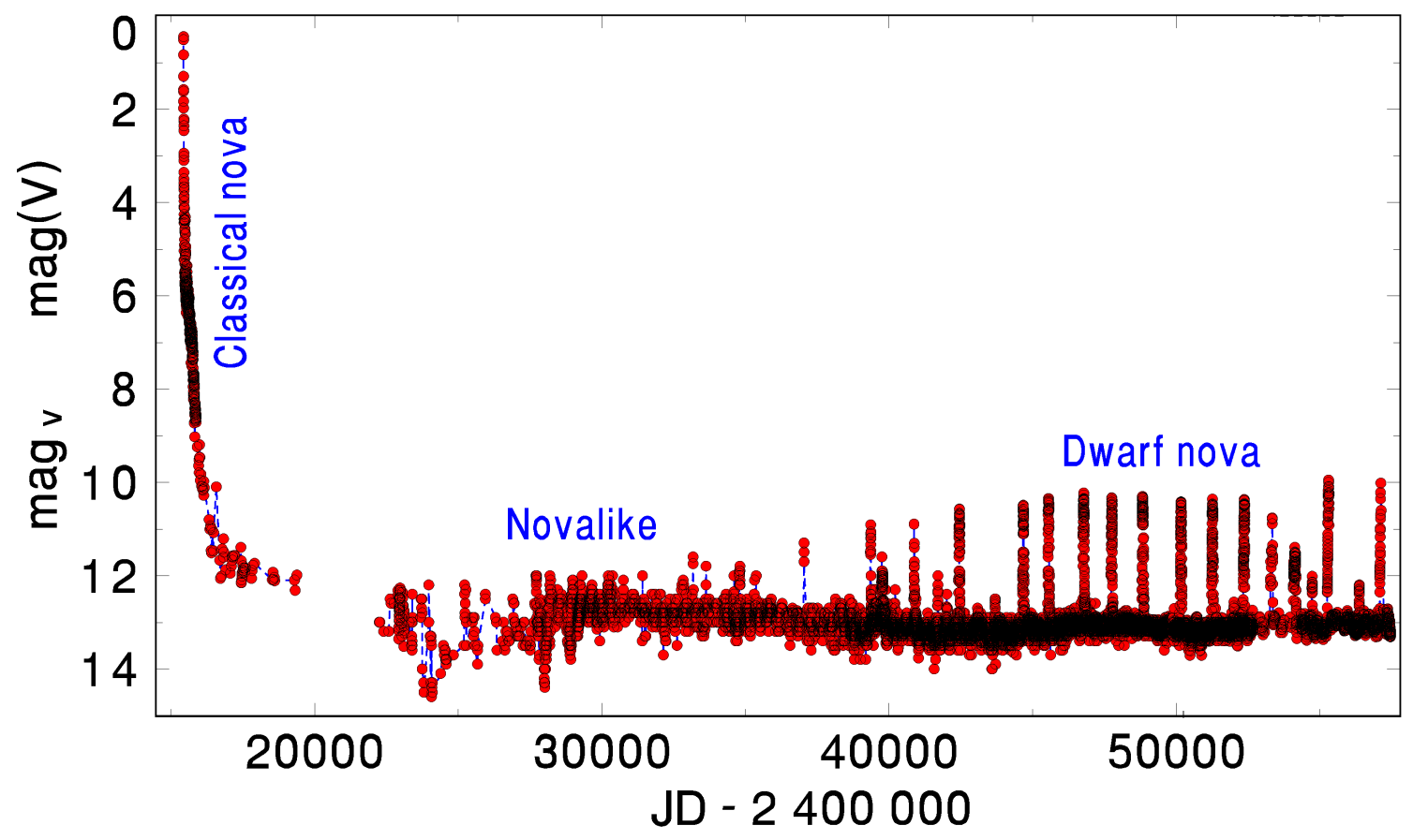

Figure 3: Long-term activity of GK Per. The type of activity changed from classical nova to novalike, and finally to dwarf nova during several tens of years. Data source: AAVSO [20].

The recent long dwarf nova outburst of GK Per, caused by a thermal-viscous instability (Fig. 4), shows a large discrepancy between the profiles in the optical (thermal emission) and hard X-ray band (bremsstrahlung). Because GK Per is an intermediate polar [47], accretion from the disk occurs onto the polar caps of the WD, not via boundary layer. According to [50,51], the spin modulation suggests that the mass accretion occurred onto the polar caps (not onto boundary layer) even during this outburst in Fig. 4. This spin modulation was not due to absorption [51]. Structural changes of the accretion regions at the poles of the WD are necessary for explaining this saturation of X-ray intensity in the time of the largest mass inflow through the disk. Already [19] showed that an increase of accretion onto the polar caps in such outburst of GK Per leads to an increase of hard $\mathrm{X}$-ray luminosity and to a further hardening of the X-ray spectrum of the bremsstrahlung emission, but this absorption is not able do explain the plateau in Fig. 4b. We already showed in [38] that the saturation and even a dip of the hard X-ray intensity occurs during the strongest outbursts of GK Per. The most recent event in Fig. 4 confirms this finding and shows that the properties of the outburst largely differ for the individual, even neighboring events. This emphasizes the necessity to examine an ensemble of outbursts in such systems like GK Per.

\subsection{Serpentis}

$\mathrm{X}$ Ser appears to be another example of the change of the mechanisms of activity with time. This CV is an old classical nova which exploded in 1903 [9, 31]. Fig. 5 shows that X Ser displays strong activity even in quiescence, many years after the end of the nova explosion. A largeamplitude irregular activity in quiescence (Fig. 5, see also [17] for CCD $V$ band light curve between 


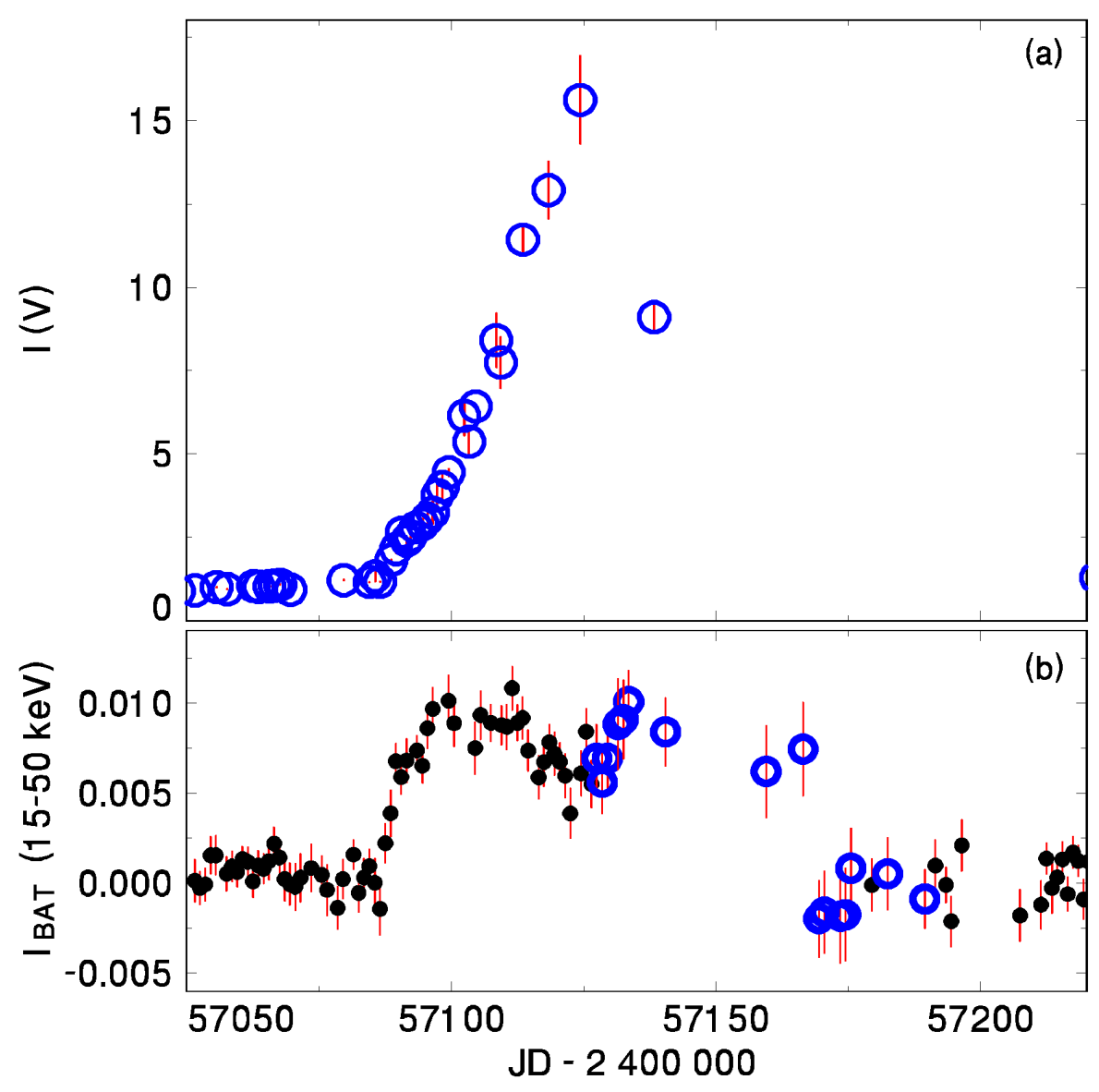

Figure 4: A recent long outburst of GK Per. (a) The optical band (one-day means, intensity scale) (AAVSO data [20]). (b) One-day means of the BAT/Swift (15-50 keV) observations (data source: [22]). The open circles have the larger error bars, but they still define the profile of the X-ray light curve of the outburst very well.

1992 and 1997) can suggest operation of several mechanisms: thermal-viscous disk instability, fluctuations of $\dot{m}_{\mathrm{T}}$.

A recent brightening of $\mathrm{X}$ Ser in Fig. $6 \mathrm{~b}$ has the decaying rate which correspond to Bailey relation [1] of dwarf-nova outburst in a CV with an exceptionally long orbital period (for $P_{\text {orb }}=$ $1.498 \mathrm{~d}$ [43]). Also the rising branch of this brightening speaks in favor of a dwarf nova outburst. In addition, a transient brightening in the DASCH photographic data (Fig. 6a) has the profile, the amplitude $(\sim 2.5 \mathrm{mag})$, and the decaying rate similar to the event in Fig. $6 \mathrm{~b}$. This suggests that $\mathrm{X}$ Ser gradually evolves toward isolated outbursts during quiescence. The dwarf-nova outbursts in such a system start when $T_{\text {eff }}$ of the WD (and/or the accretion rate) decrease after the classical nova explosion sufficiently to enable the transitions of the disk between the hot and cool states. The outbursts and gradual fluctuations in Fig. 5 and [17] can suggest that the accretion disk of X Ser fluctuated between a thermally unstable regime and a residing on the hot branch of this instability. 


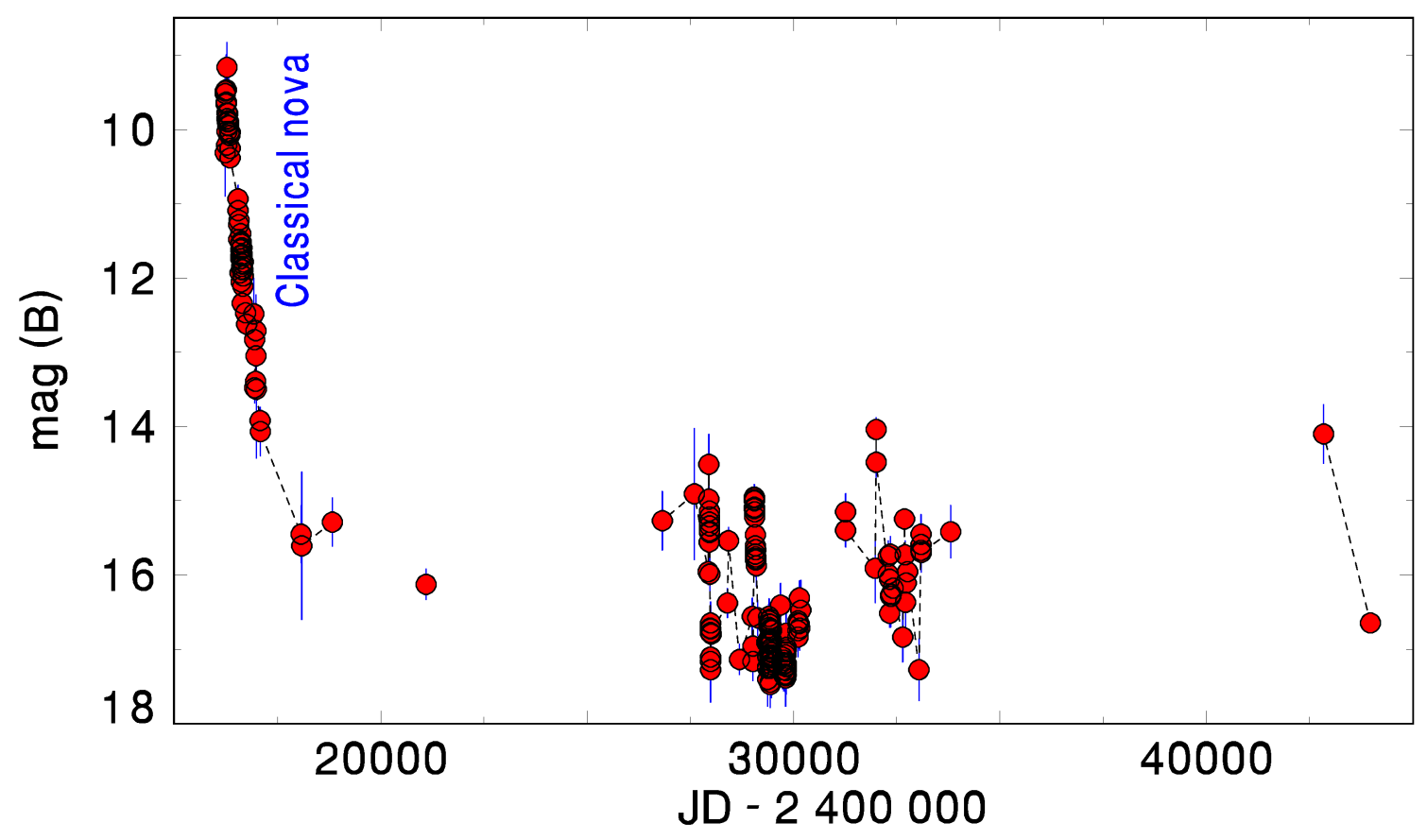

Figure 5: Long-term activity of $X$ Ser. The type of activity changed from classical nova to novalike, and finally to dwarf nova during several tens of years. Data source: DASCH photographic observations (blue light).
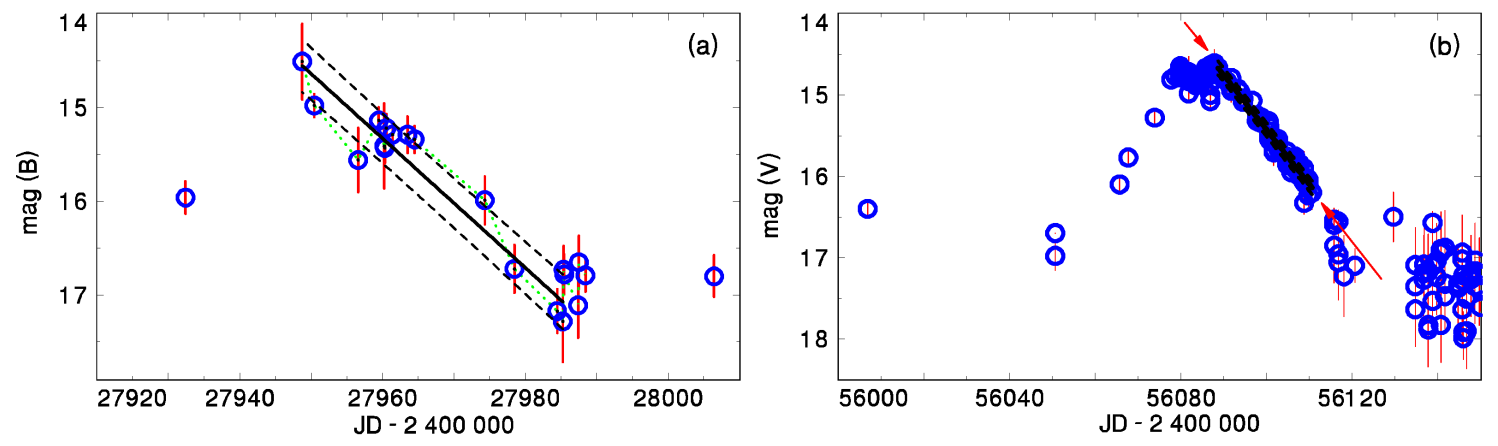

Figure 6: Dwarf nova-type outbursts of X Ser. (a) DASCH photographic light curve. (b) CCD $V$ band AAVSO data [20]. The decaying branch and the standard deviation of a linear fit are marked for both events.

\section{Supersoft X-ray sources}

Supersoft X-ray sources, in which both the steady-state hydrogen burning and the accretion processes contribute to the luminosity, often display strong long-term activity in various spectral regions. According to [29], a very intense X-ray emission of the very hot atmosphere of the WD (with the spectrum similar to black body) in the band with $E<1.5 \mathrm{keV}$ is produced. Some of these systems show an anti-correlation of the optical and soft X-ray intensities in the recurrent X-ray ON and OFF states (e.g. [30]). They were observed to became intense soft X-ray emitters in the 
recurrent optical low-state episodes. This is ascribed to a large sensitivity of the very soft X-ray emission to absorption intrinsic to the source (e.g. accretion disk) [12] and changes of the radius of the hydrogen burning envelope on the WD [30].

V Sge $\left(P_{\text {orb }}=0.514195 \mathrm{~d}[16]\right)$ is a transient supersoft X-ray source, and can be used as a laboratory for investigation of the time variations of generating the observed luminosity. The optical luminosity, generated mainly by the accretion disk, is in antiphase with X-ray luminosity (mainly from the hydrogen burning) [12] on super-orbital timescale. The transitions are usually considerably faster than the duration of the state (Fig. 7a). The two-sided moving averages with the filter half-width $Q=220 \mathrm{~d}$ (method described in [3]) of the time evolution of the standard deviation of brightness are a measure of the scatter caused by the long-term activity (the recurring high and low states) of V Sge (Fig. 7b). This approach shows that the depth of the high- and low-state episodes varies on timescale of several epochs.

The WWZ-transform (method of [10]) shows that the transitions between the high and low states of V Sge are cyclic within some time segments (Fig. 7c), which suggests that the individual states are dependent on each other. This means that the occurrence of each high- an low-state episode is dependent on the properties of the system which produce a series of the state transitions. Please note that even in the optical low state, the optical luminosity (hence $\dot{m}_{\mathrm{T}}$ ) of V Sge is still considerably larger than what is possible for operation of a thermal-viscous instability (Fig. 1). In the model of [13], the decreases of $\dot{m}_{\mathrm{T}}$ cause that the disk shrinks and the wind from the WD stops, which leads to a transition to the optical low state of V Sge. We note that our previous analysis [36] showed that the activity of V Sge displays intervals of the suppressed brightness variations (flat segments), interchanging with intervals of the pronounced changes (active segments - mostly the series of the high and low states). The series of the high and low states in Fig. 7 suggests that $\mathrm{V}$ Sge was in active segment. The moving averages of the time evolution of the standard deviation of brightness, $\sigma$, in Fig. $7 \mathrm{~b}$ shows that the series of the states is not homogeneous. The minimum of $\sigma$ (a measure of the scatter caused by the long-term activity (the recurring high and low states)) coincides with the break in the cycle in Fig. 7c.

\section{Polars}

Polars are CVs with so strongly magnetized WD that no accretion disk can form. The dominant emission thus comes from several processes in small accretion region(s) on the WD [23]. The light curve of the prototype of polars, AM Her, in very hard X-rays (15-35 keV) in the Palermo BAT Catalogue and database at INAF-IASF Palermo [35, 7] is displayed in Fig. 8.

A comparison of the X-ray and optical light curves shows that although both the optical (cyclotron (e.g. [11, 23])) and hard X-ray (bremsstrahlung (e.g. [32, 23])) luminosities increase largely in each high-state episode, the cyclotron and the bremsstrahlung luminosities are not tightly correlated. The soft X-ray excess, ascribed to thermal emission from the surface of the WD heated by the impact [23], is beyond the band observed by most monitors.

These variations suggest changes of the dimensions and structure of the cyclotron emitting region(s), not only the changes of the mass accretion rate onto the WD. The activity during a decline (much less steep than the state transition) from an initial very bright peak near JD 2455100 can be explained by a gradual evolution of the conditions in stratified shock regions in a single high 

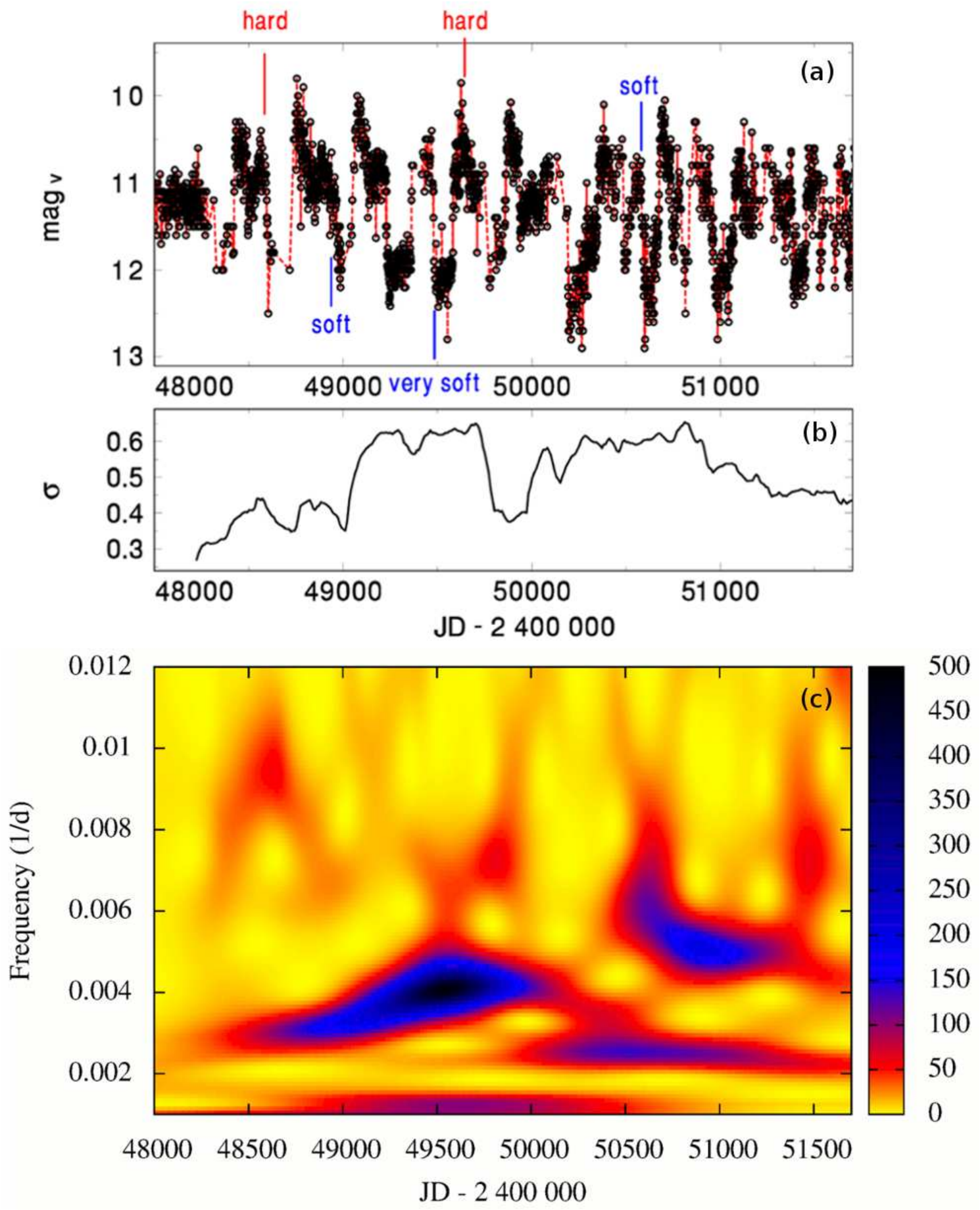

Figure 7: (a) A segment of the long-term activity of V Sge (one-day means of AAVSO and AFOEV data). The times of the ROSAT X-ray data from [12] are marked. (b) Moving averages of the time evolution of the standard deviation of brightness (a measure of the scatter caused by the long-term activity (the recurring high and low states)). (c) Cycles in the high-low state transitions (the WWZ-transform: method of [10]). 


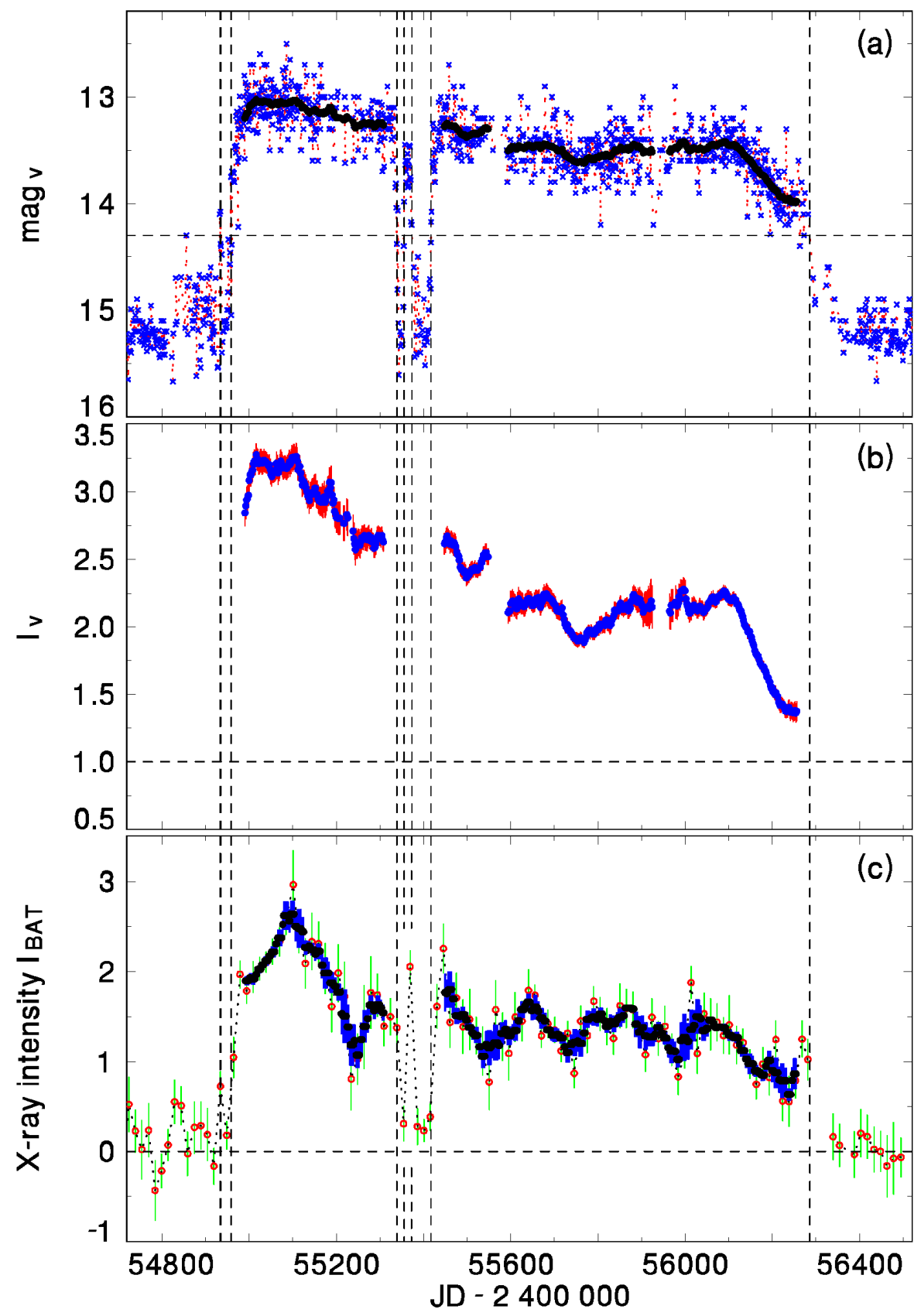

Figure 8: (a) Activity of AM Her in the optical band (magnitude scale) (AAVSO data [15, 20]). The crosses represent the one-day means. Closed circles denote the moving averages and their standard errors in the high states (distant more than 30 days from the transition). The dashed vertical lines represent the times of crossing the level of 14.3 mag. (b) Optical light curve of the high states in the intensity scale (the fit from panel (a)). The intensity is set to unity at $14.3 \mathrm{mag}$. (c) BAT/Swift data $(15-35 \mathrm{keV})\left(\left[\mathrm{ct} \mathrm{s}^{-1} \mathrm{pixel}^{-1}\right] \times 10^{4}\right)$ (open circles). For the high-state data, closed circles represent the moving averages with their standard errors. 
state episode (modeled by [6]). A cycle in the intensity of the bremsstrahlung emission during the high state may be caused by the changes of the profile of the mass stream (model of [41]). Each high-state episode is a complex phenomenon in the history of accretion in this system. More details are given in [39].

\section{Conclusions}

We show that CVs are important laboratories for investigation of accretion processes. We emphasize that $\mathrm{CVs}$ work as the systems strongly variable with time. Their activity often displays episodic events, discrete levels of intensity, and/or discrete phenomena (e.g. outbursts, low state episodes). Not any two such events are the same even in a given CV system.

This activity strongly depends on the type of the CV. Cycles of activity (often on timescale of months to years) are common. Strong structural changes of the emitting regions with time occur with the transitions between the states of activity. The character of the long-term activity (and operation of the emission processes) largely depends on the mass inflow into the lobe of the WD, and on the strength of the magnetic field of this WD. Investigation of interplay of various emitting regions (and emission processes) in various states of activity is very important.

Multifrequency observations are important for investigating the activity of CVs caused by the accretion processes. This requires a co-operation of the ground-based segment (e.g. observing thermal emission) and satellites, observing in the X-ray band (e.g. bremsstrahlung, thermonuclear burning on the WD). Only this approach enables to investigate the structural changes during this activity. We also show that some important features of activity of CVs were observed only in the optical band prior to the beginning of the X-ray astronomy. The modern observing technologies are able to observe only the consequences of such events.

Acknowledgments This study was supported by grant 13-39464J provided by the Grant Agency of the Czech Republic. This research has made use of the observations provided by the Palermo BAT Catalogue and database operated at INAF-IASF Palermo, and public data from Swift/BAT transient monitor provided by the Swift/BAT team. I acknowledge The DASCH project at Harvard, partially supported from NSF grants AST-0407380, AST-0909073, and AST-1313370. This research has also made use of the observations from the AAVSO International database (USA) and the AFOEV database (France). I thank the variable star observers worldwide. This publication also made use of the data from the Northern Sky Variability Survey created jointly by the Los Alamos National Laboratory and University of Michigan. The NSVS was funded by the Department of Energy, the National Aeronautics and Space Administration, and the National Science Foundation. I used the code developed by Dr. G. Foster and available at ww.aavso.org/winwwz.

\section{References}

[1] J. Bailey, JBAA 86, 30 (1975).

[2] K. Beuermann, et al., $A \& A$ 419, 291 (2004).

[3] P. J. Brockwell, \& R. A. Davis, Time series: Theory and Methods, Springer-Verlag New York Inc. 1987. 
[4] D. J. Collins, \& P. J. Wheatley, MNRAS 402, 1816 (2010).

[5] F. A. Cordova, \& K. O. Mason, MNRAS 206, 879 (1984).

[6] M. Cropper, et al., MNRAS, 293, 222 (1998).

[7] G. Cusumano, et al., A\&A 510, A48 (2010).

[8] J. E. Drew, et al., MNRAS 338, 401 (2003).

[9] H. W. Duerbeck, \& W. C. Seitter, LNP 369, 165 (1990).

[10] G. Foster, AJ 112, 1709 (1996).

[11] B. T. Gänsicke, et al., A\&A 372, 557 (2001).

[12] J. Greiner, \& A. van Teeseling, $A \& A$ 339, L21 (1998).

[13] I. Hachisu, \& M. Kato, ApJ 598, 527 (2003).

[14] J.-M. Hameury, et al., MNRAS 298, 1048 (1998).

[15] A. A. Henden (2014), Observations from the AAVSO International Database, http://www.aavso.org

[16] G. H. Herbig, et al., ApJ 141, 617 (1965).

[17] R. K. Honeycutt, et al., AJ 115, 2527 (1998).

[18] R. Hudec, BAICz 32, 93 (1981).

[19] M. Ishida, et al., MNRAS 254, 647 (1992).

[20] S. Kafka (2016), Observations from the AAVSO International Database, http://www.aavso.org

[21] E. Körding, et al., Sci 320, 1318 (2008).

[22] H. A. Krimm, et al., ApJS 209, 14 (2013).

[23] E. Kuulkers, et al., in Compact stellar X-ray sources. Ed. by Walter Lewin \& Michiel van der Klis. Cambridge Astrophysics Series, No. 39, Cambridge University Press 2006.

[24] Christopher W. Mauche, ApJ 610, 422 (2004).

[25] P. J. Meintjes, et al., ApJ 401, 325 (1992).

[26] P. J. Meintjes, et al., ApJ 434, 292 (1994).

[27] K. L. Page, et al., A\&A 570, A37 (2014).

[28] J. Patterson, \& J. C. Raymond, ApJ 292, 535 (1985).

[29] R. Popham, \& R. Di Stefano, LNP 472, 65 (1996).

[30] K. Reinsch et al., A\&A 354, L37 (2000).

[31] F. A. Ringwald, et al., MNRAS 281, 192 (1996).

[32] R. E. Rothschild, et al., ApJ 250, 723 (1981).

[33] F. Sabbadin, \& A. Bianchini, A\&AS 54, 393 (1983).

[34] M. R. Schreiber, et al., $A \& A$ 362, 268 (2000).

[35] A. Segreto, et al., $A \& A$ 510, id.A47 (2010).

[36] V. Šimon, \& J. A. Mattei, A\&AS 139, 75 (1999). 
[37] V. Šimon, $A \& A$ 406, 613 (2003).

[38] V. Šimon, A\&A 575, id.A65, 8 pp. (2015).

[39] V. Šimon, Ap\&SS 361, 235 (2016).

[40] J. Smak, AcA 34, 161 (1984).

[41] H. S. Stockman, \& A. F. Lubenow, (1987). Ap\&SS 131, 607 (1987).

[42] P. Szkody, AJ 108, 639 (1994).

[43] J R. Thorstensen, \& C. J. Taylor, MNRAS 312, 629 (2000).

[44] E. P. J. van den Heuvel, et al., A\&A 262, 97 (1992).

[45] B. Warner, MNRAS, 227, 23 (1987).

[46] B. Warner, Cataclysmic Variable Stars, Cambridge Univ. Press, Cambridge 1995.

[47] M. G. Watson, et al., MNRAS 212, 917 (1985).

[48] P. J. Wheatley, et al., MNRAS 345, 49 (2003).

[49] W. M. Wolf, et al., ApJ, 777, 136 (2013).

[50] Yuasa, T., et al., MNRAS, 459, 779 (2016).

[51] P. Zemko, et al., arXiv:1603.03286v1 [astro-ph.SR] (2016). 